\title{
Simultaneous Routing and Power Allocation using Location Information
}

\author{
Rocco Di Taranto and Henk Wymeersch \\ Department of Signals and Systems, Chalmers University of Technology, Gothenburg, Sweden \\ E-mail: $\{$ taranto, henkw\}@ chalmers.se
}

\begin{abstract}
To guarantee optimal performance of wireless networks, simultaneous optimization of routing and resource allocation is needed. Optimal routing of data depends on the link capacities which, in turn, are determined by the allocation of communication resources to the links. Simultaneous routing and resource allocation (SRRA) problems have been studied under the assumption that (global) channel state information (CSI) is collected at a central node. This is a drawback as SRRA depends on channels between all pairs of nodes in the network, thus leading to poor scalability of the CSI-based approach. In this paper, we first investigate to what extent it is possible to rely solely on location information (i.e., position of nodes) when solving the SRRA problem. We also propose a distributed heuristic based on which nodes can locally adjust their rate based on the local CSI. Our numerical results show that the proposed heuristic achieves near-optimal flow in the network under different shadowing conditions.
\end{abstract}

\section{INTRODUCTION}

There are many ways in which communication networks can move information from source to destination nodes, especially in the presence of multiple paths between nodes. The fundamental idea motivating optimal design is to select operating points as solution of well-defined optimization problems, which, if the optimization criteria (i.e., objective function and constraints) are chosen appropriately, guarantee the best possible network operation in a well-defined sense. Extensive research efforts (see [1]-[3] and references therein) have been dedicated to optimal design of wireless networks and results in this field include architectural insights and protocol design.

A major drawback shared by most of the optimal design research is the reliance on global channel state information (CSI), i.e., the optimal design problem depends on the channels between all pairs of terminal in the network. While availability of global CSI is plausible in certain situations, it is unlikely to hold if time-varying fading channels are taken into account, or when very large networks are considered. More recent work assumes that only local CSI is available at the nodes, which implies that operating variables of each terminal are selected as functions of the channels linking the terminal with neighboring nodes [4]. This leads [4] to propose an algorithm that, with limited amount of message passing among nodes and small computational cost, converges almost surely in an ergodic sense, to the optimal solution.

Outside the context of optimal design, the problem of scalability and lack of CSI was also tackled by the networking community. Relevant in our context is geographic routing, wherein only local CSI is available, and where each node is aware of its own geographic location, the location of its immediate neighbors, and the location of the destination [5]. Under these assumptions, the source sends data to the destination through intermediate nodes, which are selected according to a distance-to-destination criterion. By doing so, packets can be routed to the destination without knowledge of the network topology or a prior route discovery. Despite these attractive properties, geographic routing is sub-optimal and does not easily allow multiple routes towards a destination. Nevertheless, next generation wireless devices, e.g., 4G handsets, already have the capability to self-localize and provide location information with high level of accuracy everywhere and anytime [6]. We believe that this additional information can be harnessed in the design of routing protocols, in a similar way as it was shown to benefit the physical layer [7]-[9].

In this paper, we investigate how and to what extent location information can aid routing capabilities in wireless networks, in the context of optimal design. Our approach is centralized, but scales better than conventional CSI-based solutions. We assume that nodes report their position to a central planner, which makes routing and resource allocation decisions for all nodes. We compare four routing strategies: (i) the benchmark approach from [10], [11], relying on full CSI; (ii) a variation of [10], [11], where nodes report only their position to the central planner and links that are found to be in outage are discarded; (iii) an improved version of (ii), where the maximum flow is computed based on the CSI of the active links; (iv) a decentralized heuristic to approximate the maximum flow, based on local CSI and local rate adaptation; and (v) greedy geographic routing. Our contributions are as follows:

- We propose the use of location information in simultaneous routing and resource allocation (SRRA) problems, due to the lower overhead and limited performance degradation;

- We show that simple heuristics can mitigate the impact of outages due to mismatch between assumed channels (based on path loss) and actual channels (based on path loss and fading), by relying on local communication.

\section{SyStem MODEL}

In this section, we describe the network, flow, and communication model [10], [11]. Our aim is to maximize the rate 


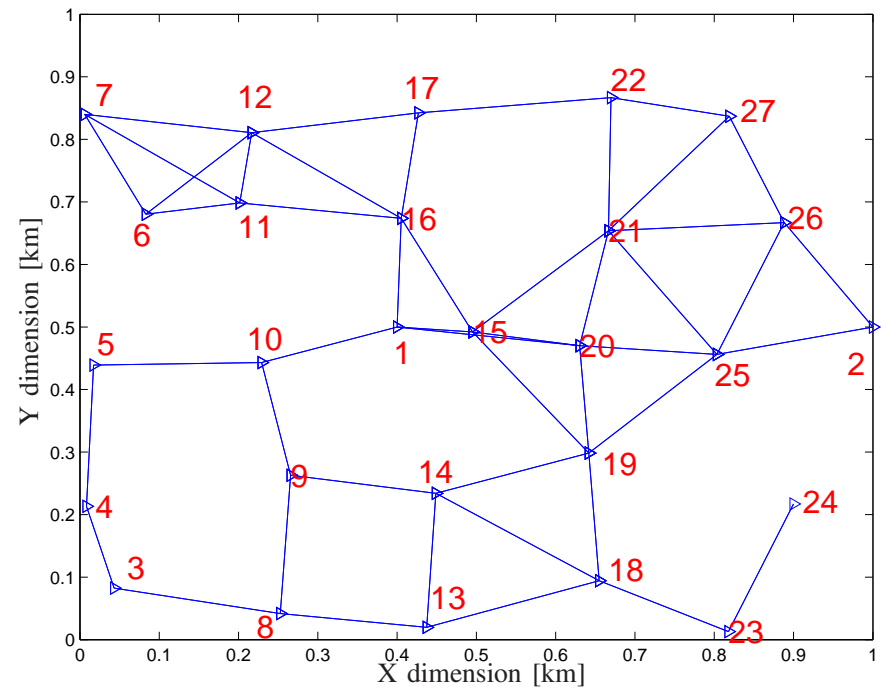

Fig. 1. Target scenario with one destination (node 1), one source (node 2), and 28 relay nodes. There are 94 directed links between nodes.

from a single source to a single destination over a number of intermediate nodes.

\section{A. Network Model}

We consider a network with $N$ nodes, indexed by $\{1,2, \ldots, N\}$. Node 1 represents the destination node, while node 2 is designated the source node. Intermediate nodes serve as relays. Communication links exist between nodes and are modeled as ordered pairs $(i, j), i \neq j$, indicating that a nonzero rate can be supported from node $i$ to node $j$. This rate depends on the resources allocated to this link as well as the channel from node $i$ to $j$ (more on this in Section II-C). The links are labeled $\{1,2, \ldots, L\}$. We denote the $N \times L$ incidence matrix by $\mathbf{A}$, so that $a_{n l}=1$ when link $l$ ends in node $n$, $a_{n l}=-1$ when link $l$ starts in node $n$, and $a_{n l}=0$ otherwise.

\section{B. Flow Model}

The flow model aims to capture the flow from source to destination, and the conservation of flow at intermediate nodes. To this end, we introduce a source vector $\mathbf{s} \in \mathbb{R}^{N}$, with $s_{2} \geq 0$ denoting the flow generated by the source for the destination, $s_{1}=-s_{2}$, and $s_{n}=0$ for $n \neq\{1,2\}$. At any relay node $n \neq\{1,2\}$ the incoming flow must equal the outgoing flow. To model this, we introduce $\mathbf{x} \in \mathbb{R}^{L}$, where $x_{l} \geq 0$ denotes the flow over link $l$, which can be related to $s_{n}$ by

$$
\mathbf{a}_{n}^{\mathrm{T}} \mathbf{x}=s_{n}, \quad \forall n,
$$

where $\mathbf{a}_{n}^{\mathrm{T}}$ is the $n$-th row of $\mathbf{A}$.

\section{Link Model}

We assume links do not interfere, as would occur in, e.g., a time-division multiple access or frequency-division multiple access scenario. With every link $l$ we associate a limited set of resources $r_{l}$, which may comprise transmit power and signaling bandwidth. The rate over link $l$ depends not only on the allocated resources, but also on the physical propagation channel. The channels are modeled to comprise path loss and shadow fading. Denoting the distance between the nodes on link $l$ as $d_{l}$, the channel (power) gain thus becomes

$$
h_{l}=10^{-\mathrm{PL}\left(d_{l}\right) / 10} 10^{Z_{l} / 10},
$$

with log-normal shadowing $Z_{l} \sim N\left(0, \sigma_{\mathrm{z}}^{2}\right)$ and path loss $\operatorname{PL}\left(d_{l}\right)=\operatorname{PL}\left(d_{0}\right)+10 \eta \log _{10}\left(d_{l} / d_{0}\right)$, in which $\eta>0$ is the path loss exponent and $\mathrm{PL}\left(d_{0}\right)$ is the line-of-sight path loss at reference distance $d_{0}$. Overall, the rate $x_{l}$ over a link $l$ is limited by the capacity, which depends on $r_{l}$ and $h_{l}$ (as well as noise power):

$$
0 \leq x_{l} \leq \phi_{l}\left(r_{l}, h_{l}\right)
$$

where we limit $\phi_{l}\left(r_{l}, h_{l}\right)$ to be concave and monotonically increasing in $r_{l}$. Finally, the set of resources available to each link are limited by a vector $\mathrm{g}$, modeled through a constraint of the form $\mathbf{F r} \preceq \mathrm{g}$, which is able to capture per-node power constraints as well as per-link bandwidth constraints, for suitable $\mathbf{F}$ and $\mathbf{g}$. $\mathbf{F}$ has the same size as the incidence matrix $\mathbf{A}$ and its elements are given by $F_{n l}=\max \left\{0, A_{n l}\right\}$.

\section{THE SRRA PROBLEM}

Combining the network flow model and communication model described in the previous two sections, we now formulate the maximum utility version of the SRRA problem, assuming an objective function that is concave as a function of the source rate $s_{2}$ and of the transmitting power $r_{l}$ at all nodes:

$$
\begin{array}{ll}
\text { maximize } & f\left(s_{2}\right)-\eta \sum_{l=1}^{L} r_{l} \\
\text { subject to } & \mathbf{A x}=\mathbf{s} \\
& 0 \leq x_{l} \leq \phi_{l}\left(r_{l}, h_{l}\right) \\
& \mathbf{F r} \preceq \mathbf{g}, \mathbf{0} \preceq \mathbf{r},
\end{array}
$$

where the optimization variables are $\mathbf{x}, \mathbf{s}, \mathbf{r}$. The second term in the objective function penalizes the expense of unnecessary power in the network, e.g., along loops, which do not contribute to the primary objective $f(\cdot)$. The constant $\eta$ is selected appropriately to balance the importance of the second term in the utility function. The problem (4) is a convex optimization problem and can be solved efficiently by, for example, general interior point methods. Moreover, in the above model, the matrices $\mathbf{A}$ and $\mathbf{F}$ are sparse and highly structured, so efficient algorithms can be developed by exploiting the problem structure. In this paper, we study the centralized SRRA problem, where the channel state information $h_{l}$ is obtained either exactly from beaconing with channel estimation (referred to as gain based GB-SRRA) or approximately from positioning system (referred to as location based LB-SRRA). The solution to SRRA will be denoted by $\mathbf{x}^{*}, \mathrm{~s}^{*}, \mathrm{r}^{*}$. 


\section{A. Gain-based SRRA}

The gain-based SRRA (GB-SRRA) operates as follows. The $N$ nodes exchange high-power beaconing signals to estimate the channels $h_{l}$. We will assume channel estimation is perfect. These channel estimates are then collected by the SRRA unit, which solves the problem (4) and yields to corresponding maximal rate from source to destination, denoted by $s_{\mathrm{GB}}^{*}$. While optimal in our setting, GB-SRRA has a large overhead during the beaconing phase, as $\mathcal{O}\left(N^{2}\right)$ channel estimates may need to be collected, which is prohibitive (in terms of transmission power and delay) in large networks with thousands of nodes. This is the main motivation for LB-SRRA.

\section{B. Location-based SRRA}

The location-based SRRA (LB-SRRA) avoids $\mathcal{O}\left(N^{2}\right)$ beaconing overhead and extracts channels through position information. LB-SRRA operates as follows: The $N$ nodes send their positions to the SRRA unit. We assume position estimation is perfect. The SRRA unit can then infer approximate channel $\tilde{h}_{l}=10^{-\mathrm{PL}\left(d_{l}\right) / 10}$, which is generally different from $h_{l}$ but requires only $\mathcal{O}(N)$ overhead. The SRRA unit solves the problem (4) with the approximate channels (i.e., $\tilde{h}_{l}$ instead of $h_{l}$ ) and yields to corresponding maximal rate from source to destination, denoted by $s_{\mathrm{LB}, \mathrm{PL}}^{*}$. Note that generally $s_{\mathrm{LB}, \mathrm{PL}}^{*} \neq s_{\mathrm{GB}}^{*}$, since when $\sigma_{\mathrm{z}}^{2}>0, h_{l} \neq \tilde{h}_{l}$ for most links. When the resources are allocated to the nodes and communication commences, the allocated links are estimated locally, so that CSI becomes available on a small subset of links. When $\tilde{h}_{l} \neq h_{l}$, there will be mismatches between $x_{l}^{*}$ and $\phi_{l}\left(r_{l}^{*}, h_{l}\right)$. This implies that some links are in outage (i.e., when $\left.x_{l}^{*}>\phi_{l}\left(r_{l}^{*}, h_{l}\right)\right)$ while other links may be underutilized (i.e., $\left.x_{l}^{*}<\phi_{l}\left(r_{l}^{*}, h_{l}\right)\right)$.

We consider three different approaches to deal with this mismatch:

1) Remove links in outage: In the first case, links in outage (those for which $x_{l}^{*}>\phi_{l}\left(r_{l}^{*}, h_{l}\right)$ ) are disconnected. The resulting rate will be denoted by $s_{\mathrm{LB} \text {,out }}^{*} \leq$ $\min \left(s_{\mathrm{GB}}^{*}, s_{\mathrm{LB}, \mathrm{PL}}^{*}\right)$, and can be obtained through classical min-cut/max-flow methods [13].

2) Centralized max-flow: In the second case, for the given resource allocation (i.e., $r_{l}^{*}$ ), the maximum flow between source and destination is computed using the maxflow/mincut algorithm, i.e., each link carries a traffic that is smaller or equal than its capacity $\phi_{l}\left(r_{l}^{*}, h_{l}\right)$. The resulting rate is denoted as $s_{\mathrm{LB}}^{*}$.

3) Distributed approximate max-flow: In the third case, nodes operate as follows. Nodes first compute $x_{l}=$ $\phi_{l}\left(r_{l}^{*}, h_{l}\right)$ for all incident links, based on the actual channels of the scheduled links. Every node $n$ then determines $I(n)$, the total incoming flow (i.e., the sum of the incoming flows $x_{l}$ ) and $O(n)$, the total outgoing flow (i.e., the sum of the incoming flows $x_{l}$ ). The distributed algorithm then proceeds as follows: (i) pick a random node, say $n$, for which $I(n) \neq O(n)$; (ii) if $I(n)<O(n)$, reduce all the outgoing flows $x_{l}$ with a proportion $I(n) / O(n)$; if $I(n)>O(n)$, reduce all the incoming flows $x_{l}$ with a proportion $O(n) / I(n)$; (iii) recompute $I(n)$ and $O(n)$ and go back to step (i). Due to the fact that the flows are lower-bounded by zero and that we always reduce flows, this algorithm is guaranteed to converge to a flow that can be satisfied globally. The resulting rate will be denoted by $s_{\text {heuristic }}^{*}$

\section{NumericAl RESUlTs}

\section{A. Simulation Setup}

We consider a randomly generated wireless network with 30 nodes uniformly distributed in $1 \mathrm{~km}$ by $1 \mathrm{~km}$ square, depicted in Fig. 1. We impose a restriction on the communication range, whereby two nodes can communicate if their distance is smaller than 250 meters. This avoids weak channels that can support only negligible rates and still leads to a strongly connected network with 94 links (47 links between nodes, every link is bi-directional). Node 1 is selected as destination, node 2 as source, and the remaining nodes can act as relays. The channel is modeled according to (2), with shadowing variance in the range $\sigma_{\mathrm{z}}^{2} \in[0,4]$ [12]. The resources $\mathbf{r}$ are considered to be transmit powers over different links, with a per-node power constraint, so that $\mathrm{Fr} \preceq \mathrm{g}$ simplifies to

$$
\sum_{l: a_{n l}=-1} r_{l} \leq P_{n, \text { tot }}, \quad \forall n,
$$

where $P_{n \text {,tot }}$ is the power available to node $n$, and here normalized to unity. The receivers at the end of the links are subject to independent additive white Gaussian noise with variance $\sigma_{l}^{2}$, modeled to be uniformly distributed in the interval $[0.01,0.1]$. We describe the capacity $\phi_{l}\left(r_{l}, h_{l}\right)$ of link $l$ with the classical Shannon capacity formula, which satisfies the conditions in Section II-C:

$$
\phi_{l}\left(r_{l}, h_{l}\right)=\log _{2}\left(1+\frac{r_{l} h_{l}}{\sigma_{l}^{2}}\right) .
$$

Our aim is to maximize the rate, so as source utility function, we can choose any monotonically increasing function in $s_{2}$. We selected $f\left(s_{2}\right)=\log s_{2}$ and $\eta=10^{-3}$. Finally, to solve (4), we used CVX in MATLAB [14] and averaged results over 100 Monte Carlo runs.

\section{B. Discussion}

We first consider LB-SRRA and quantify the occurrence of outages (i.e., $x_{l}^{*}>\phi_{l}\left(r_{l}^{*}, h_{l}\right)$ from Section III-B). When $\sigma_{\mathrm{z}}^{2}=0$ there are no outages, so $s_{\mathrm{LB}}^{*}=s_{\mathrm{LB}, \text { out }}^{*}=s_{\mathrm{GB}}^{*}$. As soon as $\sigma_{\mathrm{z}}^{2}>0$, approximately $50 \%$ of the links will be in outage, since the constraint (6) is always tight. These outages will cause a significant reduction in flow, so that $s_{\mathrm{LB}, \text { out }}^{*} \ll$ $s_{\mathrm{GB}}^{*}$.

Fig. 3-4 show, for a specific set of channel realizations in the network of Fig. 1 and for $\sigma_{\mathrm{z}}^{2}=1$, how the traffic is routed from source node 2 to destination node 1 . In each of these figures, the thickness of each link is roughly proportional to the associated flow variable (note that since the constraint (6) is always tight, the thickness is also proportional to the power 


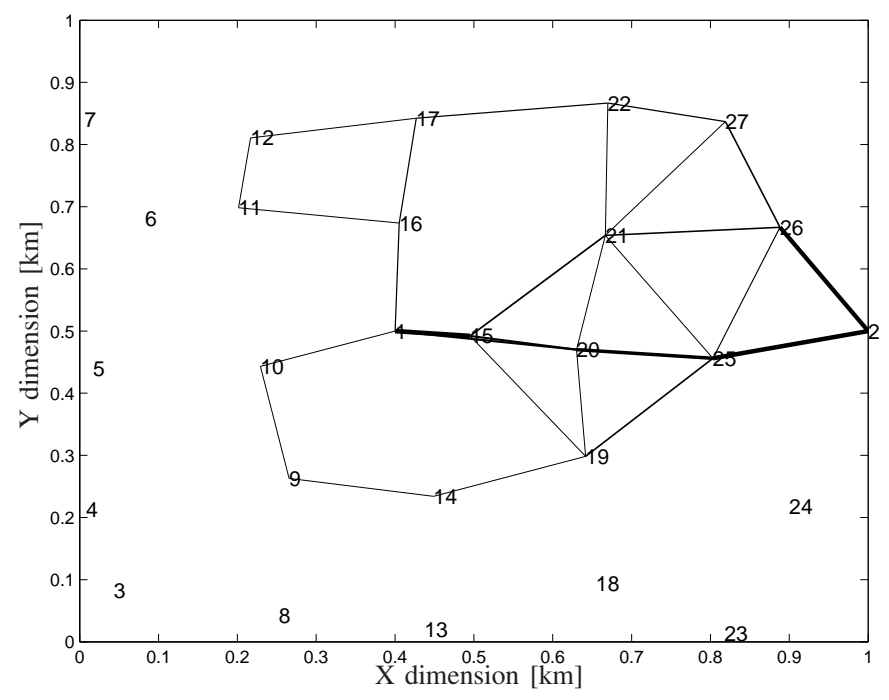

Fig. 2. Flow from source node 2 to destination node 1 with GB-SRRA.

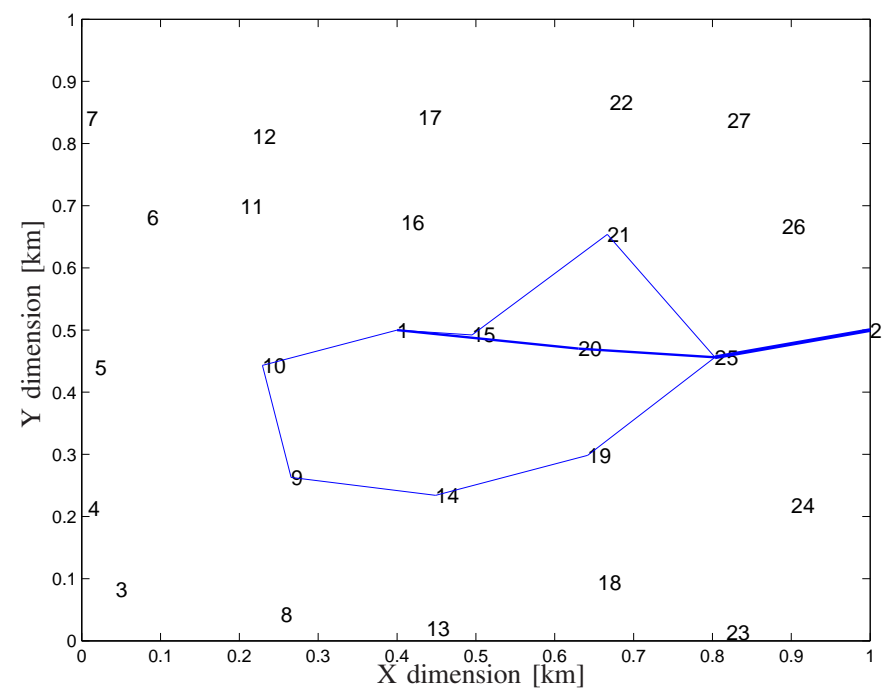

Fig. 3. Flow from source node 2 to destination node 1 with LB-SRRA and some links in outage.

used for that link). Fig. 3 shows the optimal routing from source node 2 to sink node 1 when full CSI is collected and benchmark solution $s_{\mathrm{GB}}^{*}$ is obtained. The effect of outages is displayed in Fig. 5, which shows routing in correspondence of the $s_{\mathrm{LB}, \text { out }}^{*}$ solution. It can be seen that a large fraction of the links are in outage and a much lower traffic goes through the network. Finally, Fig. 4 shows how the traffic is routed with LB-SRRA in conjunction with the max-flow/mincut algorithm. The total flow from the source to the sink is smaller and the set of active links is different compared to Fig. 3, although some common paths are still present. This happens because channel estimation based on location information through the path loss model does not account for shadowing.

A quantified view of the impact of these outages, and a comparison of the different approaches is offered in Fig. 2,

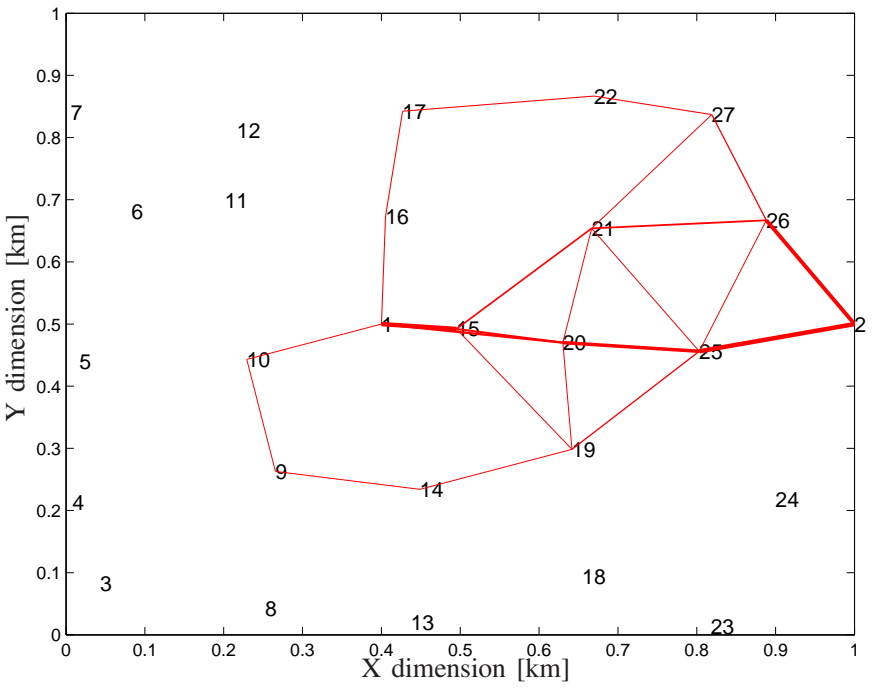

Fig. 4. Flow from source node 2 to destination node 1 with LB-SRRA and max-flow/min-cut algorithm.

which shows the total flow from source to destination, as a function of the shadowing variance. Five cases are considered: (i) gain-based SRRA (leading to solution $s_{\mathrm{GB}}^{*}$, which serves as an upper bound); (ii) location-based SRRA (leading to solution $s_{\mathrm{LB}, \text { out }}^{*}$ ); (iii) location-based SRRA and maxflow/min-cut algorithm (leading to solution $s_{\mathrm{LB}}^{*}$ ); (iv) heuristic algorithm described previously leading to solution $s_{\text {heuristic }}^{*}$; and (v) greedy geo-graphic routing (labeled as "geo-routing" in Fig. 2). Geo-routing will always use the shortest path, i.e., $2 \rightarrow 25,25 \rightarrow 20,20 \rightarrow 15$, and $15 \rightarrow 1$, and the corresponding flow will be limited by the weakest link. Case (i) leads to a flow of around 5, very slightly increasing with the amount of shadowing. This may be surprising at first sight, but has been observed previously [15] as a side-effect of independent shadowing per link. The location-based SRRA case (ii) yields high outages for $\sigma_{\mathrm{z}}^{2}>0$, which have a dramatic impact on the total flow, reducing it to with around $90 \%$. Even a few links in outage can disrupt parts of the flow, which significantly reduces overall throughput. The solution with max-flow/min-cut ( $s_{\mathrm{LB}}^{*}$, case (iii)) degrades much more gracefully with $\sigma_{z}^{2}$, with only a $20 \%$ loss in flow, even in the severest shadowing condition. The distributed max-flow approximation $s_{\text {heuristic }}^{*}$ turns out to work quite well, closely following $s_{\mathrm{LB}}^{*}$ for all values of the shadowing variance. Hence, the ability for nodes to locally estimate channel gains toward their neighbors (without reporting this information to the central planner) and adapt their rate according to the proposed algorithm, is an attractive low-complexity solution to combine good performance with low overheads. Finally, geo-routing has an acceptable performance, that is (on average) not very sensitive to the amount of shadowing. Note that our network topology from Fig. 1 favors the geo-routing protocol as intermediate nodes are deployed at regular distances between source and destination. In general, geo-routing can suffer from very low throughputs. 


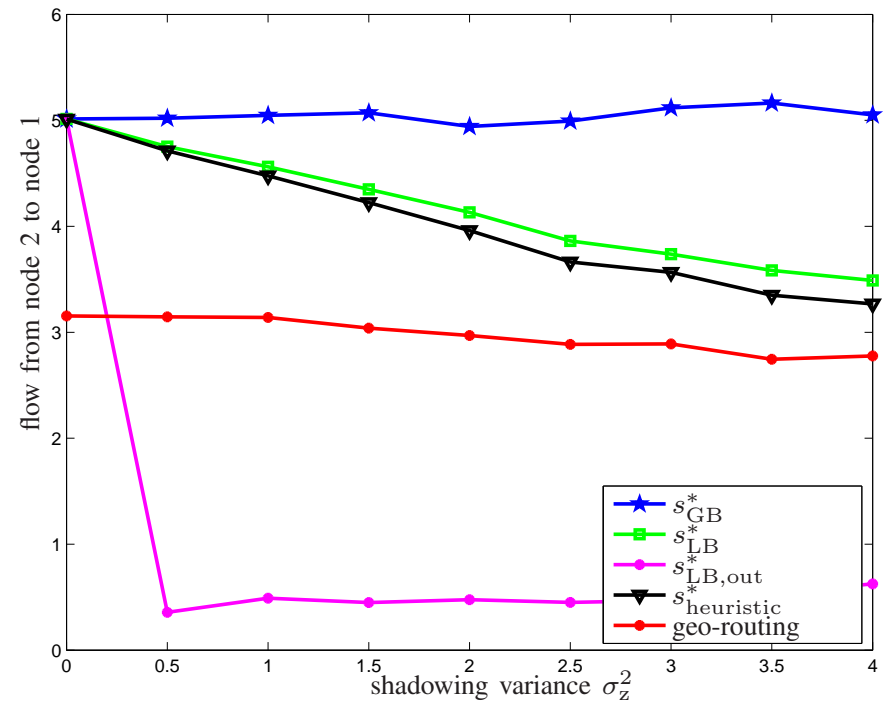

Fig. 5. System throughput for five routing strategies, as a function of the shadowing variance: the gain-based SRRA solution $\left(s_{\mathrm{GB}}^{*}\right)$, the locationbased SRRA solution with max-flow min-cut $\left(s_{\mathrm{LB}}^{*}\right)$, the location-based SRRA solution with outage $\left(s_{\mathrm{LB}, \text { out }}^{*}\right)$, the proposed heuristic $\left(s_{\text {heuristic }}^{*}\right)$ and greedy geographic routing.

\section{Conclusions}

In this paper, we have investigated to what extent it is possible to rely on location information (i.e., geographic position of nodes) when solving the simultaneous optimization of routing and power allocation. It turns out that frequent link outages limit the performance. We have proposed an heuristic based on which nodes can adjust their rate by locally estimating the CSI only toward their neighbors. Our numerical results indicate that the proposed heuristic, while significantly reducing the overhead in the network, can achieve a near-optimal flow in the network, under different shadowing conditions. A comparison with greedy geographic routing reveals that our proposed heuristic can achieve superior performance due to path diversity.

Future work will consider myopic versions of the simultaneous routing and power allocation problem with limited CSI, which are a more fair comparison to geographic routing. Based on [4], it can be conjectured that a two-hop view will achieve good performance with only localized processing.

\section{ACKNOWLEDGMENT}

This research was supported in part, by the European Research Council, under Grant No. 258418 (COOPNET). R. Di Taranto acknowledges support from the Swedish Research Council through Grant No. 2011-6864. The authors would like to thank Gunther Maday for his help in implementing the heuristic proposed in this work.

\section{REFERENCES}

[1] M. Chiang, S. H. Low, R. A. Calderbank, and J.C. Doyle, "Layering as optimization decomposition," in Proceedings IEEE, no. 1, pp. 255-312, Jan. 2007.

[2] X. Lin, N. B. Shroff, and R. Srikant, "A tutorial on cross-layer optimization in wireless networks," in IEEE J. Sel. Areas Comm., vol. 24, no. 8, pp. 1452-1463, Aug. 2006.

[3] L. Georgiadis, M. J. Neely, and L. Tassiulas, "Resource allocation and cross-layer control in wireless networks," in Found. Trends Netw., vol. 1, no.1, pp. 1-144, 2006.

[4] Y. Hu and A. Ribeiro, "Optimal Wireless Networks Based on Local Channel State Information," in IEEE Transactions on Signal Processing, vol. 60, issue 9, pp. 4913-4929, Sept. 2012.

[5] F. Cadger, K. Curran, J. Santos, and S. Moffett, "A Survey of Geographical Routing in Wireless Ad-Hoc Networks," in Communications Surveys \& Tutorials, IEEE, vol.15, no.2, pp.621-653, Second Quarter 2013.

[6] J.A. Del Peral-Rosado, J.A. Lopez-Salcedo, G. Seco-Granados, F. Zanier, and M. Crisci, "Analysis of positioning capabilities of 3GPP LTE," in Proc. ION GNSS, 2012.

[7] S. Sand, R. Tanbourgi, C. Mensing, and R. Raulefs. "Position aware adaptive communication systems," in Conference Record of the FortyThird Asilomar Conference on Signals, Systems and Computers, pp. 7377, 2009.

[8] H. Celebi and H. Arslan "Utilization of location information in cognitive wireless networks," in IEEE Wireless Communications, vol. 14, no. 4, pp. 6-13, Aug. 2007.

[9] J. J. Nielsen, T. K. Madsen, and H.P. Schwefel, "Location Based Mobile Relay Selection and Impact of Inaccurate Path Loss Parameters," in Proc. IEEE Wireless Communication and Networking Conference, pp.1-6, 613, April 2010.

[10] L. Xiao, M. Johansson, and S. Boyd, "Simultaneous Routing and Resource Allocation via Dual Decomposition", in Proceedings of 4th Asian Control Conference, pages 29-34, September 25-27, 2002, Singapore.

[11] L. Xiao, M. Johansson, and S. Boyd, "Simultaneous Routing and Resource Allocation via Dual Decomposition", in IEEE Transactions on Communications, 52(7):1136-1144, July 2004.

[12] A. Molish, Wireless Communications. Second Edition, John Wiley \& Sons Ltd, 2011.

[13] G.L. Nemhauser and A. W. Laurence, Integer and combinatorial optimization. Vol. 18. New York: Wiley, 1988.

[14] M. Grant, and S. Boyd, CVX: Matlab software for disciplined convex programming, version 2.0 beta. http://cvxr.com/cvx, September 2012.

[15] C. Bettstetter, and C. Hartmann. "Connectivity of wireless multihop networks in a shadow fading environment.", in Wireless Networks 11.5: pp. 571-579, 2005. 